\title{
Quantifying flexibility in EV charging as DR potential: Analysis of two real-world data sets
}

\author{
Chris Develder*, Nasrin Sadeghianpourhamami*, Matthias Strobbe*, and Nazir Refa ${ }^{\dagger}$ \\ ${ }^{*}$ Ghent University - iMinds, INTEC - IBCN \\ Technologiepark Zwijnaarde 15, BE-9052 Gent, Belgium \\ Email: \{cdvelder, nsadeghian, mstrobbe\}@intec.ugent.be \\ ${ }^{\dagger}$ ElaadNL \\ Utrechtseweg 310, building B42, 6812 AR Arnhem, The Netherlands \\ Email: nazir.refa@elaad.nl
}

\begin{abstract}
The increasing adoption of electric vehicles (EVs) presents both challenges and opportunities for the power grid, especially for distribution system operators (DSOs). The demand represented by EVs can be significant, but on the other hand, sojourn times of EVs could be longer than the time required to charge their batteries to the desired level (e.g., to cover the next trip). The latter observation means that the electrical load from EVs is characterized by a certain level of flexibility, which could be exploited for example in demand response (DR) approaches (e.g., to balance generation from renewable energy sources).

This paper analyzes two data sets, one from a charging-athome field trial in Flanders (about 8.5k charging sessions) and another from a large-scale EV public charging pole deployment in The Netherlands (more than 1M sessions). We rigorously analyze the collected data and quantify aforementioned flexibility: (1) we characterize the EV charging behavior by clustering the arrival and departure time combinations, identifying three behaviors (charging near home, charging near work, and park to charge), (2) we fit statistical models for the sojourn time, and flexibility (i.e., non-charging idle time) for each type of observed behavior, and (3) quantify the the potential of DR exploitation as the maximal load that could be achieved by coordinating $\mathrm{EV}$ charging for a given time of day $t$, continuously until $t+\Delta$.
\end{abstract}

\section{INTRODUCTION}

Adoption of electric vehicles (EVs) presents both challenges and opportunities for the power grid, especially for distribution system operators (DSOs). On the one hand, the charging power they need leads to an increase in the electrical demand [1], [2], since a single full electric vehicle's energy demand approximately amounts to that of a single typical residential household. On the other hand, their batteries could be used as temporary storage. To assess the expected impact on the power grid, it is thus of crucial importance to characterize the EV users in terms of the EV charging behavior: at what times do users charge their cars, for how long, etc. To fulfill the demand, one may want to exploit the flexibility that stems from sojourn times of parked EVs that are longer than the time required to (fully) charge the battery. A reasonably accurate model of that flexibility is required to realistically assess, e.g., the effectiveness of demand response (DR) strategies (for instance to balance available renewable energy sources with EV load).

This paper presents, to the best of our knowledge, the first quantitative analysis of the DR potential resulting from such flexibility, in terms of the maximal load that could be attained (or avoided) by coordinating EV charging, based on (relatively) large-scale, real-world data. The contributions of our work are:

- We present two real-world data sets, the largest of which logged over $1 \mathrm{M}$ actual EV charging sessions from real users in operational settings (Section III),

- By clustering of the observed charging sessions in terms of $\mathrm{EV}$ arrival/departure times, we derive three different types of charging behavior (Section IV-A),

- We provide statistical models (e.g., for use in simulation studies of DR) that characterize for each type of behavior both the total sojourn time (i.e., from arrival till departure) as well as the flexibility defined as idle time spent not charging (i.e., sojourn minus effective charging duration) (Section IV-B), and finally

- We propose to quantify the potential for DR exploitation of the observed flexibility in EV charging, as an upper bound for the load that could be achieved by coordination through a DR algorithm, and discuss the numerical results thereof for the real-world data sets (Section V).

Before detailing these contributions, in the next Section II we outline related work in the area of characterization of EV charging, as well as the estimation of flexibility. The overall conclusions of the paper are summarized in Section VI.

\section{RELATED WORK}

This paper can be positioned in the research on characterization of EV charging behavior. An early line of work in this area, before a reasonably wide-spread deployment of EVs had occurred in practice, comprised the development of models of driving behavior, as to establish arrival and departure patterns of cars (as well as the distance covered during the trips in between). Examples of this approach include [3] (which combines statistical characteristics of driving behavior to derive EV charging data profiles), [4] (based on extrapolations of non-EV car usage in Belgium) and [5] (which derives EV charging behavior models from non-EV driving behavior in Sweden). For other examples of attempts to derive EV charging assessments from regular car usage, 
we refer to [6]. On the other hand, our work presented here comprises actual, real-world EV usage data, and rather focuses on assessing the charging session characteristics (not only in terms of timing, but also in terms of electrical load) and especially their flexibility and the resulting demand response (DR) potential.

Thus, our data can be used to quantitatively assess the load stemming from EV charging. Several works in literature have already pursued that goal. For instance, Xydas et al. [2] use data from about $22 \mathrm{k}$ sessions, which they statistically analyze and cluster, to eventually derive the total energy demand (in $\mathrm{kWh}$ ). Similarly in spirit, Khoo et al. [1] study almost 5k sessions from an Australian field trial and establish the expected impact on the total power demand in 2032-33 for the state of Victoria. The objective of our analysis presented here rather was to quantify the flexibility of the EV load, and quantitatively study user behavior.

User modeling (not focusing on flexibility) has clearly been the subject of earlier works, including [1], [2], [7], [8]. For example, Helmus et al. [7] characterize different user types in terms of EV charging session start and end times (with associated charged energy), as well as the time between subsequent charging sessions. They distinguish different user types defined a priori, including 'residents', 'commuters', 'taxis', etc. On the other hand, Xydas et al. [2] use a purely data-driven approach to cluster observed charging sessions into distinct types of behavior. They derive aggregate models for three specific geographic areas, characterized by different clusters of "typical EV charging demand profiles" (and further study predictability of daily peak power from weather conditions). Our present work uses similar techniques, but focuses on distinguishing different types of individual user behavior (on a non-aggregated level), and without making a priori assumptions (in terms of pre-defined user types as in [7]). A similar characterization of charging session timing is presented by Kara et al. [9, Sec. 2], who also define a "flexibility" metric (i.e., the fraction of total connection time that is spent charging). Yet, they only present overall observed histograms of start/end times over all 580k nonresidential charging sessions. We will provide a more indepth analysis and identify different types of charging behavior (with different characteristics in terms of both arrival/departure times and flexibility). We further note that our data covers residential charging as well as public charging at roadside parking spots (hence, our work is complementary to fast charging station models along highways, e.g., the fluid traffic model as presented in [10]).

Aside from aforementioned works that study the characterization of charging behavior in terms of timing and duration, other works focus on complementary aspects. For example, Haidar and Muttaqi [8] zoom in on an individual charger, which they model in detail from a power engineering perspective, i.e., in terms of active/reactive power, voltage, current evolution over time. For probabilistic powerflow calculations, [11] presents a methodology of modeling the overall charging demand of PHEVs. Moving away from such more technical aspects, Franke and Krems [12] attempt to also give a "rationale" for observed behavior, i.e., the psychological dynamics that underly that behavior.

Despite the sizeable body of work on EV user modeling, we believe the investigation of EV flexibility is rather limited. Specifically, the quantification of the potential of such EV flexibility from real-world charging sessions, for exploitation in demand response (DR) schemes has largely been missing. Yet, more generally, residential electricity demand has been studied rather extensively. For instance, De Coninck et al. [13] use a bottom-up approach to characterize flexibility with cost functions, and Engels et al. adopt regression analysis based on a price elasticity matrix. Rather than cost quantification, we focus on actual $\mathrm{kWh}$ that can be shifted in time (which subsequently can be used to assess whether the resulting DR potential would be substantial).

In the latter spirit, Wattjes et al. [14] propose a universal framework to estimate the flexibility of commercial and industrial customers, using a high level model largely based on estimates (rather than accurate measurements) of flexibility in terms of power, duration and speed of activating the flexibility (mainly from lighting and cooling). Abdisalaam et al. [15] similarly estimate potential flexibility, and among others assess the potential benefit of interrupting EV charging - however, the EV data is a synthetic model based on typical car usage in The Netherlands from 2007. On the household device side (rather than EVs), studies based on real-world data include [16] (washing machines, dryers, heaters, ACs, refrigerators), [17] (heat pumps) and [18] (wet appliances).

In conclusion, we believe the existing body of work lacks (i) data-driven modeling of EV users' charging behavior including flexibility, and (ii) DR potential assessment from EVs based on large- scale, real-world data. The remainder of this paper is a first step in filling those gaps.

\section{DATA SETS}

The data analyzed in this paper was collected in two reasonably different settings, as summarized in Table I. The first data set comprises about $8.5 \mathrm{k}$ sessions from the iMove field trial in Flanders, with charging poles installed at users' homes. The second was collected by ElaadNL from public charging infrastructure deployed throughout The Netherlands, encompassing more than $1 \mathrm{M}$ sessions so far (which is a factor $\times 2$ bigger than the largest data set we are aware of [9]). The next subsections sketch the context of both projects.

\section{A. iMove field trial: Charging-at-home}

The iMove project was one of 5 projects in the Living Labs Electrical Vehicles program ${ }^{1}$ in Flanders and ran from 2012 until 2014 [19]. Its objective was to encourage EV adoption by offering a large group of users the experience of using an EV on a daily basis. In total, the field trial deployed 175 EVs and 180 charging poles. The data for our analysis was collected from a subset comprising 50 full $\mathrm{EVs}^{2}$, that were

\footnotetext{
${ }^{1}$ See http://www.livinglab-ev.be/.

${ }^{2}$ A mixture of Renault Fluence and Renault Kangoo models.
} 
Table I: Main characteristics of the two (raw) data sets.

\begin{tabular}{lcc}
\hline & iMove & ElaadNL $^{*}$ \\
\hline Period & $03 / 2012-03 / 2013$ & $01 / 2011-12 / 2015$ \\
\# Sessions & 8,520 & $1,141,849$ \\
\# Users & 134 & $53 \mathrm{k}$ \\
Car type & Full EV & Unknown mix \\
Charge point & At home & Public \\
Trip details & Yes & No \\
\hline${ }^{\dagger}$ In iMove, at any point time, up to 50 users were active \\
(since cars were reused over time). In ElaadNL, recorded \\
charging sessions are tied to a particular charging card. \\
* The results presented in this paper are based on the sessions \\
from 1 Jan.-31 Mar. 2015 ( $N=90,562)$.
\end{tabular}

reused by different users, each time for a contiguous period of about 2 months, in the period from March 2012 till March 2013. Those cars were equipped with a GPS logger to track the distance covered during all trips in between charging sessions. The charging was done at the user's home, where the project installed a charging pole equipped to log arrival and departure times, as well as the charged energy (in $\mathrm{kWh}$ ).

\section{B. Operational ElaadNL infrastructure: Public charging}

ElaadNL, along with EVnetNL, has emerged from the "Stichting e-laad"3, which established a network of around 3,000 public charging poles for EVs across The Netherlands between 2009 and 2015. Today, EVnetNL is responsible for managing the existing charging stations, in coordination with the relevant municipalities. ElaadNL on the other hand, is the knowledge and innovation center in the field of charging infrastructure in The Netherlands, providing coordination for the connections of public charging stations to the electricity grid on behalf of 6 participating distribution system operators (DSOs). ElaadNL also performs technical tests of charging infrastructure, researches and tests smart charging possibilities of EVs, and develops communication protocols for managing EV charging (e.g., as part of energy management systems that could participate in demand response programs). Data was collected between 2011 and 2015 from the operational charging poles scattered across The Netherlands. For the subsequent analysis, we took the subset of sessions from January 1 until March 31, 2015 (i.e., 90,562 sessions).

Note that in contrast to iMove, the EVs themselves in this ElaadNL data set are privately owned cars, and thus comprise a mixture of various (a priori unknown) types, and we have no information on the driving behavior.

\section{EV CHARGING BEHAVIOR MODELING}

\section{A. Clustering of charging session times}

The first question we address is: What typical behaviors exist in terms of time of arrival and departure? Figure 1 shows the individual sessions, plotted in terms of $\left(t_{\text {arr }}, t_{\text {dep }}\right)$ (to have compact graphs, we plot time as time-of-day, i.e., plot $t$ mod $24 \mathrm{~h}$ ). To cluster the data in that 2D space, we adopted

\footnotetext{
${ }^{3}$ Literally translated to English, this means "foundation e-charge".
}

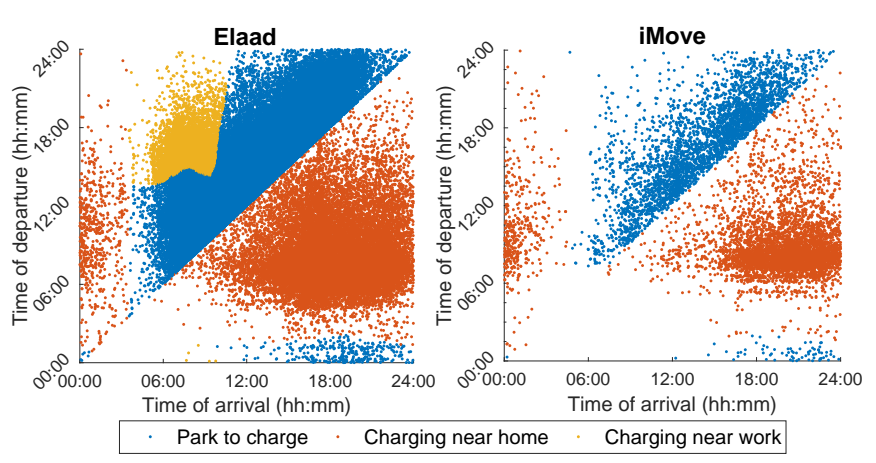

Fig. 1: Clustering the sessions in terms of EV arrival and departure times. Both $X$ - and $Y$-axis denote time-of-day (i.e., we report times as $t \bmod 24 \mathrm{~h}$ ): points below the $X=Y$ diagonal have departures on the day after the arrival or later. (Note that also some sessions plotted above the diagonal actually have departures $\geq 24 \mathrm{~h}$ after arrival; see below.)

DBSCAN [20] ${ }^{4}$ Note that DBSCAN will mark some points as "noise", and leave them unassigned to any cluster. Therefore, in a post-processing step we assigned these "noise" points to the closest of the DBSCAN clusters.

Figure 1 shows the clustering results. We find three major clusters for ElaadNL, and two for iMove. We named these clusters according to our interpretation of the observed behavior. The charge near home cluster $(59.1 \%$ of iMove sessions, $29.1 \%$ of ElaadNL) has departure times mostly in the morning, with arrivals the afternoon/evening before. This amounts to mostly nighttime charging. For the public ElaadNL data, we hypothesize these are people that live nearby the (public) charging pole, and park there car until they leave for work in the morning. The charge near work cluster $(9.4 \%$ of ElaadNL sessions) is characterized by arrivals in the morning, and departures in the evening. We assume these are people that either work near the charging pole, or at least take their car to the pole on their way to work (e.g., as part of their commute, near a train station) and leave their car there while at work. This type of behavior is absent in the iMove data set, which concerns charging at home. The park to charge cluster $(40.9 \%$ of iMove sessions, $61.5 \%$ of ElaadNL) has arrival/departure times scattered during the day, and exhibit sojourns that last not much longer than the time required to charge the battery. We hypothesize these are people that park specifically with the aim to charge the EV battery. Comparing ELaadNL to iMove, we note that the clusters seem more compact and more cleanly separated for iMove, but this is largely due to the difference in data set sizes (only $8.5 \mathrm{k}$ sessions vs. over 90k for ElaadNL).

\section{B. Statistical modeling of sojourn and idle times}

We will now further analyze the sessions within each of the behavioral clusters in terms of the durations of the sessions, and the fraction thereof that is effectively spent on charging

\footnotetext{
${ }^{4}$ We used the following parameter settings: (i) ElaadNL: Eps $=0.5$ and MinPts $=440$, and (ii) iMove: Eps $=0.7$ and MinPts $=50$.
} 


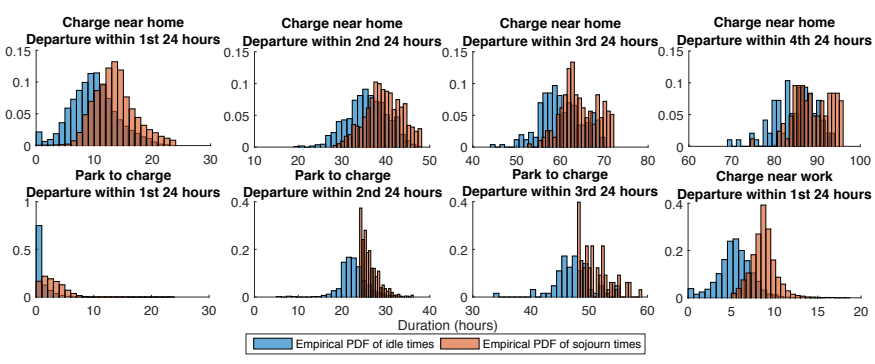

Fig. 2: Empirical distributions of total sojourn times and idle times.

the car. More formally, we define:

$$
\begin{aligned}
\text { Sojourn time } \triangleq \delta^{\text {sojourn }} & =t^{\text {depart }}-t^{\text {arrive }} \\
\text { Charging time } \triangleq \delta^{\text {charging }} & =t^{\text {end charging }}-t^{\text {start charging }} \\
\text { Idle time } \triangleq \delta^{\text {idle }} & =\delta^{\text {sojourn }}-\delta^{\text {charging }}
\end{aligned}
$$

Looking at each behavioral cluster individually, we note that a minority of sessions have sojourn times of more than $24 \mathrm{~h}$ (see Table II). We find that for such clusters, the sojourn time distribution is multimodal, where the modes correspond to subsequent days, and are well separated. We thus further divide the data into sub-clusters, based on the departure time (i.e., depending on whether it is within the first, second, etc., period of $24 \mathrm{~h}$ following the arrival). The empirical distributions of total sojourn and idle times per sub-cluster are shown in Fig. 2. For the charge near home sub-clusters, we find that the idle times lag behind with about $3 \mathrm{~h} 42 \mathrm{~min}$ (i.e., avg $\delta^{\text {charging }}=$ $3.70 \mathrm{~h}$ ) consistently over all sub-clusters. For the park to charge cluster, the same-day departure sub-cluster dominates (98.9\% of the entire cluster), and the sojourn is indeed not much longer than the time required for charging: avg $\delta^{\text {idle }} \approx$ $23 \mathrm{~min}$. For the charge near work cluster, nearly all sessions $(99.6 \%)$ have same-day departures, and have mean idle times of $5 \mathrm{~h} 18 \mathrm{~min}$ of their mean sojourn of $8 \mathrm{~h} 53 \mathrm{~min}$.

We next fit statistical distributions to the observed sojourn and idle times per sub-cluster, as reported in Table II. We first identify candidate distributions sub-cluster using a Cullen and Frey graph [21], which essentially verifies whether the kurtosis and skewness plot as observed in the empirical distributions is consistent with given distribution types. For the identified candidate distributions, we estimate their parameters using the method of matching moments. The main reason to resort to method of matching moments for parameter estimation is the existence of zeros in our data, which limits the use of maximum likelihood estimation for some distributions (e.g., Gamma). For the comparison of the fit, we compare: the empirical vs. estimated PDF plot, the empirical vs. estimated CDF plot, theoretical vs. empirical probabilities plot and theoretical vs. empirical quantiles plots. The best fit is chosen based on the smallest distance between the empirical and theoretical values in the aforementioned plots.

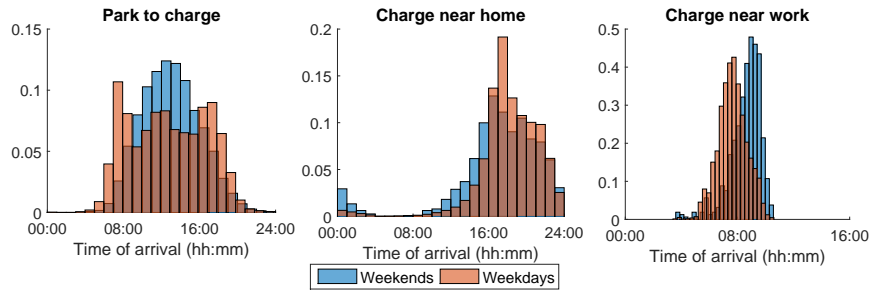

(a) Arrival time distributions

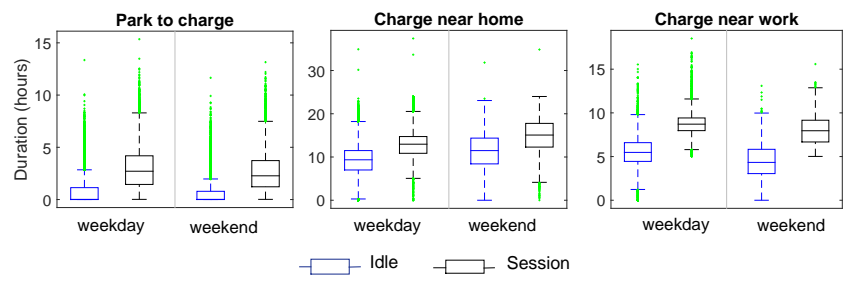

(b) Session and idle time boxplots

Fig. 3: Weekdays vs. weekends.

\section{Differences between weekdays and weekends}

Analyzing the data according to the day-of-the-week, we observe significantly different behavior between weekdays and weekend days for some of the behavioral clusters.

First, we discuss arrival times, shown in Fig. 3a. For park to charge sessions, we find that on weekdays we have peaks of arrivals in the morning, around lunch time and in the evening. In weekends, we see a smooth, unimodal distribution that peaks around noon. For charge near home, we find that arrivals are more evenly spread across the late afternoon until late at night in weekends. For charge near work, we observe a shift of arrivals with about $2 \mathrm{~h}$ to later times in weekends.

Also in terms of total sojourn time, as well as the idle portion thereof, we observe noticeable differences between weekdays and weekends, except for the park to charge behavior. For charge near home, we note longer sojourns and higher variability in weekends as opposed to weekdays (also idle times follow the same shift, implying that charging times in both cases are similar, averaging around $3.7 \mathrm{~h}$ ). For charge near work on the other hand, weekend sessions (and idle times) are on average slightly shorter, while effective charging times are a bit longer (the average charging duration increases from $3.3 \mathrm{~h}$ on weekdays to $3.6 \mathrm{~h}$ in weekends).

\section{Flexibility AND DR POTENTIAL OF EV CHARGING}

One of our main objectives at the outset this study was to quantify the demand response (DR) potential from the flexibility in the load that stems from aggregated EV charging: How much can we increase (or reduce) the power consumption at a given point in time, and for how long? That overall DR potential has two main contributing factors: the number of connected EVs, and their flexibility in terms of how much their charging can be shifted in time. The latter amounts to the flexibility present in EV charging sessions, or thus the idle times, for which we already have fit a statistical model (see Fig. 2). The first, i.e., the number of EVs that are present 
Table II: Fitted distributions for total sojourn and idle times

\begin{tabular}{|c|c|c|c|c|c|c|c|c|}
\hline \multirow[b]{2}{*}{ Cluster } & \multirow[b]{2}{*}{$\begin{array}{c}\text { Sub-cluster } \\
\text { departures }\end{array}$} & \multirow[b]{2}{*}{ Fraction } & \multicolumn{3}{|c|}{ Sojourn time $\left(\delta^{\text {sojourn }}\right)$} & \multicolumn{3}{|c|}{ Idle time $\left(\delta^{\text {idle }}\right)$} \\
\hline & & & Distr. & $\begin{array}{l}\text { Normalized distr. } \\
\text { parameters* }\end{array}$ & $\begin{array}{l}{[\min , \max ]} \\
\text { (hours) }\end{array}$ & Distr. & $\begin{array}{l}\text { Normalized distr. } \\
\text { parameters* }\end{array}$ & $\underset{\text { (hours) }}{[\min , \max ]}$ \\
\hline $\begin{array}{l}\text { Park to } \\
\text { charge } \\
(61.5 \%)\end{array}$ & $\begin{array}{l}\text { in } 1^{\text {st }} 24 \mathrm{~h} \\
\text { in } 2^{\text {nd }} 24 \mathrm{~h} \\
\text { in } 3^{\text {rd }} 24 \mathrm{~h}\end{array}$ & $\begin{array}{c}98.9 \% \\
0.9 \% \\
0.1 \%\end{array}$ & $\begin{array}{c}\text { Beta } \\
\text { Gamma } \\
\text { Gamma }\end{array}$ & $\begin{array}{c}\alpha=1.91, \beta=14.22 \\
\alpha=1.24, \beta=6.40 \\
\alpha=1.40, \beta=5.01\end{array}$ & $\begin{array}{c}{[0.02,23.91]} \\
{[24.00,36.11]} \\
{[48.01,59.93]}\end{array}$ & $\begin{array}{c}\text { Beta } \\
\text { Logistic } \\
\text { Logistic }\end{array}$ & $\begin{array}{c}\alpha=0.31, \beta=10.04 \\
\mu=0.64, s=0.06 \\
\mu=0.62, s=0.08\end{array}$ & $\begin{array}{c}{[0,23.66]} \\
{[5.05,32.35]} \\
{[34.21,55.11]}\end{array}$ \\
\hline $\begin{array}{c}\text { Charge } \\
\text { near home } \\
(29.1 \%)\end{array}$ & $\begin{array}{l}\text { in } 1^{\text {st }} 24 \mathrm{~h} \\
\text { in } 2^{\text {nd }} 24 \mathrm{~h} \\
\text { in } 3^{\text {rd }} 24 \mathrm{~h} \\
\text { in } 4^{\text {th }} 24 \mathrm{~h}\end{array}$ & $\begin{array}{l}95.4 \% \\
3.3 \% \\
0.8 \% \\
0.3 \%\end{array}$ & $\begin{array}{c}\text { Logistic } \\
\text { Beta } \\
\text { Beta } \\
\text { Beta }\end{array}$ & $\begin{array}{l}\mu=0.56, s=0.08 \\
\alpha=2.59, \beta=1.95 \\
\alpha=2.44, \beta=1.61 \\
\alpha=2.91, \beta=1.39\end{array}$ & $\begin{array}{c}{[0.02,23.99]} \\
{[28.13,47.95]} \\
{[52.84,72.00]} \\
{[74.75,95.86]}\end{array}$ & $\begin{array}{l}\text { Normal } \\
\text { Normal } \\
\text { Normal } \\
\text { Normal }\end{array}$ & $\begin{array}{c}\mu=0.42, \sigma^{2}=0.17 \\
\mu=0.57, \sigma^{2}=0.16 \\
\mu=0.57, \sigma=0.21 \\
\mu=0.64, \sigma^{2}=0.20\end{array}$ & $\begin{array}{c}0,23.53] \\
{[19.37,47.86]} \\
{[47.25,70.00]} \\
{[69.05,93.73]}\end{array}$ \\
\hline $\begin{array}{c}\text { Charge } \\
\text { near work } \\
(9.4 \%)\end{array}$ & in $1^{\text {st }} 24 \mathrm{~h}$ & $99.6 \%$ & Logistic & $\mu=0.27, s=0.06$ & {$[5.00,18.52]$} & Logistic & $\mu=0.35, s=0.07$ & {$[0,15.54]$} \\
\hline
\end{tabular}

* The parameters of distributions are the following: (i) Normal: mean $\mu$ and variance $\sigma^{2}$, (ii) Beta: shape parameters $\alpha$ and $\beta$, (iii) Gamma: shape $\alpha$ and rate $\beta$, (iv) Logistic: location parameter $\mu$ and scale parameter $s$. Note that the parameter values are reported for fits of normalized data, i.e., durations are rescaled per sub-cluster as $\delta_{\text {normalized }}=\frac{\delta-\min }{\max -\min }$.

at a given time, straightforwardly follows from the arrival and departure times (which are summarized in Fig. 1). We combine these factors and quantitatively define the DR potential as follows:

$P_{\mathrm{FLEX}}(t, \Delta) \triangleq$ the maximal amount of power that a DR algorithm can trigger to either (i) consume for the complete time interval $[t, t+\Delta]$, or (ii) avoid using at all, by shifting it to another time (entirely outside of $[t, t+\Delta]$ ).

Adopting the $\delta^{\cdots}$ symbols introduced earlier in eq. (1)-(3), and denoting sessions $s \in S$, with charging power $P_{s}$, this leads to the following more formal definition:

$$
P_{\mathrm{FLEX}}(t, \Delta)=\sum_{s \in S_{\mathrm{FLEX}}(t, \Delta)} P_{s}
$$

where

$$
\begin{aligned}
& S_{\mathrm{FLEX}}(t, \Delta)= \\
& \left\{s \in S:[t, t+\Delta] \subset\left[t_{s}^{\text {arrive }}, t_{s}^{\text {depart }}\right]\right. \\
& \\
& \left.\wedge \delta_{s}^{\text {charging }} \geq \Delta \wedge \delta_{s}^{\text {idle }} \geq \Delta\right\} .
\end{aligned}
$$

Indeed, (5) expresses that the sessions to account for in the FLEX power (i) completely overlap the interval $[t, t+\Delta]$, (ii) have a charging duration of at least $\Delta$ (so we can sustainably consume $P_{s}$ for the entire $[t, t+\Delta]$ ), and (iii) their idle time is at least $\Delta$ (so we can move away the power consumption entirely outside of $[t, t+\Delta]$ if the DR algorithm wants to).

Figure 4 plots the results of this DR potential assessment, for weekdays and weekend days separately, and for values of $\Delta$ ranging from $15 \mathrm{~min}$ to $4 \mathrm{~h}$ (= $240 \mathrm{~min})$. To illustrate how to read this graph: consider the $P_{\mathrm{FLEX}}$ result for charge near home, at $t=7 \mathrm{am}$ on a weekend day, for $\Delta=15 \mathrm{~min}$, where we see that $P_{\text {FLEX }}(7 \mathrm{am}, 15 \mathrm{~min}) \approx 750 \mathrm{~kW}$. This means that at $7 \mathrm{am}$ on a weekend morning, a DR algorithm could potentially achieve an additional load of $750 \mathrm{~kW}$ (on top of, e.g., the residential base load stemming from all household consumption except the EV) by appropriately scheduling the

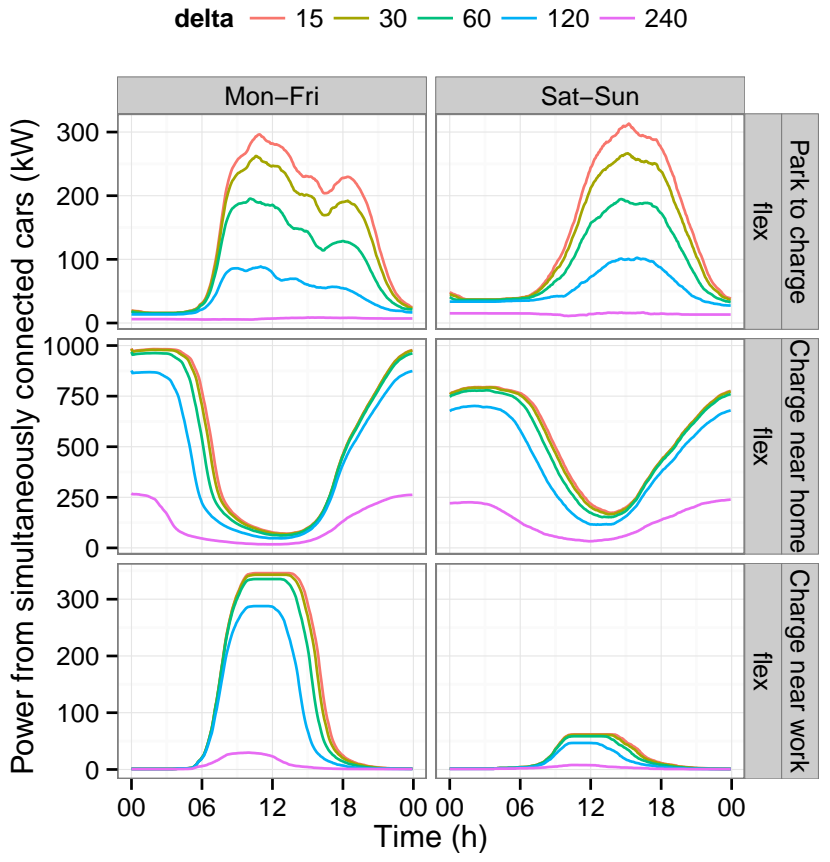

Fig. 4: Demand response potential: The power $P_{\mathrm{FLEX}}(t, \Delta)$ that we can choose to either fully consume, or not at all, during the complete time interval $[t, t+\Delta]$, by coordinating EV charging.

charge near home EVs, and maintain that extra load until at least 7:15 am. (Note that effectively exploiting this particular flexibility would likely impact the remaining potential at later times, e.g., at 7:30 am - yet, the $P_{\text {FLEX }}$ curves do not consider this, and hence are upper bounds for the DR potential.)

Comparing the three behavior clusters, we note that the charge near home cluster mainly provides flexibility at night time - as expected - although it spreads out over a larger portion of the day during weekends. The charge near work is complementary and provides flexibility during daytime, but mostly on weekdays. The smaller park to charge cluster also exhibits daytime flexibility, which is fairly uniformly spread 
over the day during the week, and more peaked around early afternoon in the weekends; we also note its shorter flexibility duration (with more distinctly lower $P_{\mathrm{FLEX}}$ when $\Delta$ is higher).

\section{Vi. Conclusions}

We collected data from real-world EV charging sessions, for which we (i) provided a comprehensive data analysis, and (ii) quantified the DR potential in terms of the sustained power consumption that we can achieve by coordinating EV charging. We found three distinct behaviors through clustering, and provided an intuitive rationale for each of them. The charge near home behavior concerns arrivals mostly in the late afternoon and evening, with departures mostly in the morning and hence long sojourn times (averaging over $15 \mathrm{~h}$ and sometimes spanning multiple days). This leads to night-time flexibility mostly, which spreads out a bit in the weekend. The charge near work cluster likely has people parking their car at work, or at commute points (e.g., near a train station), with arrivals in the early morning, and departures in the late afternoon. Sojourns are fairly long (average around $9 \mathrm{~h}$ ), and resulting flexibility occurs during daytime and mostly on weekdays. The last park to charge behavior exhibits shorter sojourns (slightly more than $3 \mathrm{~h}$ ) and limited flexibility per session. The aggregated flexibility occurs throughout the day on weekends, and is more peaked around early afternoon on weekends. In terms of statistical models, we found that total sojourn times in our data set can be accurately modeled by Beta, Logistic or Gamma distributions (depending on the cluster) and the idle times by Beta, Logistic or Normal distributions. Next steps include constructing generative models (e.g., for simulation studies), following an approach similar to [22].

\section{ACKNOWLEDGMENTS}

Work in this paper was supported in part by the Ministry of Science of Flanders through the iMove project, funded via the Agency for Innovation by Science and Technology (IWT). We thank ElaadNL for providing the data and relevant insights on EV charging in The Netherlands. Special thanks goes out to Stan Janssen (ElaadNL) for preparing the raw data for analysis.

\section{REFERENCES}

[1] Y. B. Khoo, C.-H. Wang, P. Paevere, and A. Higgins, "Statistical modeling of Electric Vehicle electricity consumption in the Victorian EV Trial, Australia," Transport. Res. Part D-Transport. Environ., vol. 32, pp. 263-277, Oct. 2014.

[2] E. Xydas, C. Marmaras, L. M. Cipcigan, N. Jenkins, S. Carroll, and M. Barker, "A data-driven approach for characterising the charging demand of electric vehicles: A UK case study," Appl. Energy, vol. 162, pp. 763-771, Jan. 2016.

[3] I. Lampropoulos, G. Vanalme, and W. Kling, "A methodology for modeling the behavior of electricity prosumers within the smart grid," in Proc. 1st IEEE PES Innovative Smart Grid Technol. Conf. Eur. (ISGT Europe 2010), Gothenburg, Sweden, 11-13 Oct. 2010, pp. 1-8.
[4] K. Clement-Nyns, K. Van Reusel, and J. Driesen, "The consumption of electrical energy of plug-in hybrid electric vehicles in Belgium," in Proc. 2nd Eur. Ele-Drive Transportation Conf. (EET 2007), Brussels, Belgium, Jun. 2007, pp. 1-10.

[5] P. Grahn, K. Alvehag, and L. Sder, "PHEV utilization model considering type-of-trip and recharging flexibility," IEEE Trans. Smart Grid, vol. 5, no. 1, pp. 139-148, Jan. 2014

[6] P. Grahn, "Electric vehicle charging modeling," $\mathrm{PhD}, \mathrm{KTH}$, Sweden, 2014.

[7] J. Helmus and R. van den Hoed, "Unraveling user type characteristics: Towards a taxonomy for charging infrastructure," in Proc. 28th Int. Electric Vehicle Symp. and Exhibition (EVS 28), Goang, Korea, 3-6 May 2015, pp. 1211-1226.

[8] A. M. A. Haidar and K. M. Muttaqi, "Behavioral characterization of electric vehicle charging loads in a distribution power grid through modeling of battery chargers," IEEE Trans. Ind. Appl., vol. 52, no. 1, pp. 483-492, Jan. 2016.

[9] E. C. Kara, J. S. Macdonald, D. Black, M. Brges, G. Hug, and S. Kiliccote, "Estimating the benefits of electric vehicle smart charging at non-residential locations: A data-driven approach," Applied Energy, vol. 155, pp. 515-525, Oct. 2015. [Online]. Available: http://www.sciencedirect.com/science/article/pii/S0306261915007059

[10] S. Bae and A. Kwasinski, "spatial and temporal model of electric vehicle charging demand," IEEE Trans. Smart Grid, vol. 3, no. 1, pp. 394-403, Mar. 2012.

[11] G. Li and X. P. Zhang, "Modeling of plug-in hybrid electric vehicle charging demand in probabilistic power flow calculations," IEEE Trans. Smart Grid, vol. 3, no. 1, pp. 492-499, Mar. 2012.

[12] T. Franke and J. F. Krems, "Understanding charging behaviour of electric vehicle users," Transp. Res. Pt. F - Traffic Psychol., vol. 21, pp. 75-89, Nov. 2013.

[13] R. De Coninck and L. Helsen, "Bottom-up quantification of the flexibility potential of buildings," in Proc. 13th Int. Conf. Building Physics Perf. Simul. Assoc. (Building Simulation 2013), Chambery, France, 2528 Aug. 2013, pp. 3139-3146.

[14] F. Wattjes, S. Janssen, and J. Slootweg, "Framework for estimating flexibility of commercial and industrial customers in Smart Grids," in Proc. 4th IEEE PES Innovative Smart Grid Technol. Eur. (ISGT Europe 2013), Lyngby, Denmark, 6-9 Oct. 2013, pp. 1-5.

[15] A. Abdisalaam, I. Lampropoulos, J. Frunt, G. Verbong, and W. Kling, "Assessing the economic benefits of flexible residential load participation in the Dutch day-ahead auction and balancing market," in Proc. 9th Int. Conf. Eur. Energy Market (EEM 2012), Florence, Italy, 10-12 May 2012, pp. $1-8$.

[16] M. Pipattanasomporn, M. Kuzlu, S. Rahman, and Y. Teklu, "Load profiles of selected major household appliances and their demand response opportunities," IEEE Trans. Smart Grid, vol. 5, no. 2, pp. 742-750, Mar. 2014.

[17] K. Kouzelis, Z. Tan, B. Bak-Jensen, J. Pillai, and E. Ritchie, "Estimation of residential heat pump consumption for flexibility market applications," IEEE Trans. Smart Grid, vol. 6, no. 4, pp. 1852-1864, Jul. 2015.

[18] W. Labeeuw, J. Stragier, and G. Deconinck, "Potential of active demand reduction with residential wet appliances: A case study for Belgium," IEEE Trans. Smart Grid, vol. 6, no. 1, pp. 315-323, Jan. 2015.

[19] S. Heyvaert, T. Coosemans, J. Van Mierlo, and C. Macharis, "Living lab electric vehicles flanders (Belgium): The influence of testing an EV on the general appreciation of electric mobility," in Proc. 27th Int. Electric Vehicle Symp. and Exhibition (EVS 27), Barcelona, Spain, 17-20 Nov. 2013, pp. 1-7.

[20] M. Ester, H.-P. Kriegel, J. Sander, and X. Xu, "A density-based algorithm for discovering clusters in large spatial databases with noise," in Proc. 2nd Int. Conf. Knowledge Discovery and Data Mining (KDD 1996), Portland, OR, 2-4 Aug. 1996, pp. 226-231.

[21] A. C. Cullen and H. C. Frey, Probabilistic techniques in exposure assessment: A handbook for dealing with variability and uncertainty in models and inputs. Springer, 1999.

[22] N. Sadeghianpourhamami, M. Strobbe, and C. Develder, "Real-world user flexibility of energy consumption: Two-stage generative model construction," in Proc. 31st ACM/SIGAPP Symp. Applied Computing (SAC 2016), Pisa, Italy, 4-8 Apr. 2016. 